Reprod. Nutr. Dévelop., 1980, 20 (4 B), 1393-1399.

\title{
Absorption des substances dissoutes dans le milieu, des particules et des produits de la digestion extracellulaire chez Actinia equina (Cnidaria, Actiniaria)
}

par M. VAN PRAËT.

Laboratoire de Biologie des Invertébrés marins et Malacologie, Muséum national d'Histoire naturelle, 57, rue Cuvier, 75005 Paris.

Summary. Absorption of substances dissolved in the sea-environment, particles and extracellular digestion products in Actinia equina (Cnidaria, Actiniaria).

The results of nutrition experiments with glucose ${ }^{14} \mathrm{C}$, leucine ${ }^{3} \mathrm{H}$, amino acids ${ }^{14} \mathrm{C}$, cyanophyceae ${ }^{14} \mathrm{C}$ and lipids have permitted me to enlarge our present knowledge of actinian nutrition.

Ectodermal absorption. - Glucose and amino acids dissolved in the sea-environment were rapidly absorbed by the ectoderm. The multiple tentacles of Actinia and their cell microvilli enlarged the ectodermal surface. There is no preoral digestion, and the macromolecules were not absorbed since the ectoderm does not possess phagocytic cells.

Digestion and endodermal absorption. - Macromolecules, particles and prey were carried into the coelenteron. The prey were enclosed in the convoluted lower part of mesenteries where they were divided into fragments and molecules by enzymes secreted by the zymogen cells of the mesenterial filaments. The macromolecules, particles and prey fragments (up to a few micrometers) produced by this extracellular digestion, or collected in the environment, were absorbed by the phagocytic cells. The lipids were pinocyted by the same cells concentrated in some parts of the endoderm, but the smallest molecules (carbohydrates and amino acids) were immediately absorbed by the mesenterial filament cells in contact with the prey.

The transfer (in both directions) of the different absorbed substances between the ectoderm and the endoderm was slow. Glucose seemed to diffuse through the mesoglea, while the amino acids and the macromolecules would be transferred by the mobile cells of the mesoglea.

\section{Introduction.}

L'absorption des substances dissoutes dans le milieu est une capaciłé générale des animaux marins (Jørgensen, 1976). Chez les actinies les auteurs considèrent que celleci s'effectue au niveau de l'ectoderme (Chia, 1972) et principalement de celui des tentacules (Schlichter, 1975). Nous avions néanmoins constaté une importante absorption d'acides aminés au niveau d'une région endodermique : celle des enteroïdes (Van Praët, 1978). Nous avons donc cherché à préciser les sites d'absorption en fonction de la taille, de la nature des substances fournies et de la méthode expérimentale suivie. 
L'apparition au cours du temps, des molécules marquées, ou de produits de leur catabolisme, dans des régions non digestives nous amène à formuler des hypothèses sur les processus de transfert chez ces animaux dépourvus de système circulatoire.

\section{Matériel et méthodes.}

Nous avons utilisé différents composés marqués au carbone 14 ou au tritium. Leur localisation est révélée sur les coupes semifines des tissus après coulage d'une émulsion autoradiographique llford K2 ou K5. Des lipides ont également été utilisés. Ils sont directement décelables en microscopie électronique sur les coupes ultrafines de tissus ayant subi une postfixation osmique.

Les incubations durent $2 \mathrm{~h}$. Elles sont effectuées dans de l'eau de mer de synthèse fraîchement préparée et contenant la substance testée. Les animaux ou les tissus, sont ensuite placés dans des bains répétés d'eau de mer de synthèse et sont fixés après des temps de chasse de $2 \mathrm{~h}$ à 6 jours.

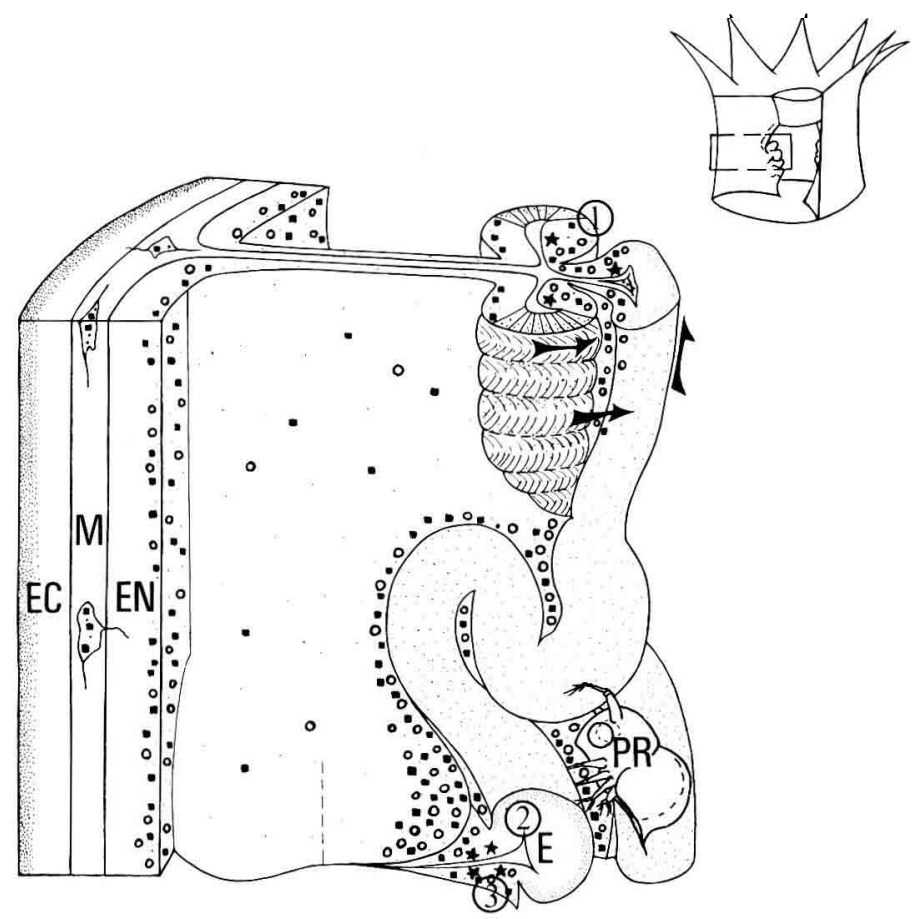

FIG. 1. - Schéma d'une portion de cavité entérique où sont récapitulées les zones d'absorption en fonction de la taille des substances.

$\because$ zone d'absorption des molécules dissoutes ;

"- zone d'absorption des macromolécules;

oo zone d'absorption des particules ayant jusqu'à quelques micromètres ;

courants d'eau sortant de la cavité entérique au nivecu des entéroïdes trilobés ;

$\star$ zone de stockage des lipides ;

$E$ : entéroïde ; $E C$ : ectoderme ; $E N$ : endoderme ; $M$ : mésoglée ; $P R$ : proie ;

(1) (2) (3) zones correspondant aux photographies 1,2 et 3 . 
Substances utilisées ef méthodes de mise en confact arec les tissus des actinies : D-glucose ${ }^{14} \mathrm{C}-1$ : activité spécifique $50 \mathrm{mCi} / \mathrm{mMole}$; concentration finale $1 \mu \mathrm{Mole} / \mathrm{ml}$. Les actinies sont immergées dans ce milieu;

L-leucine ${ }^{3} \mathrm{H}-3,4,5$ : activité spécifique $1 \mathrm{Ci} / \mathrm{mMole}$; concentration finale $0,02 \mu \mathrm{Mole} / \mathrm{ml}$. Un lot d'actinies est immergé et dans un second lot $1 \mathrm{ml}$ de cette solution est injecté dans la cavité entérique de chaque actinie ;

Hydrolysat de protéines ${ }^{14} \mathrm{C}-\mathrm{U}$ (acides aminés et glucides aminés) : activité spécifique $1,5 \mathrm{mCi} / \mathrm{mg}$; concentration finale $7 \mu \mathrm{g} / \mathrm{ml}$. Des actinies entières et des pelotes mésentériques isolées par dissection sont immergées dans ce milieu.

Cyanophycées ${ }^{14} \mathrm{C}-\mathrm{U}, 0,05 \mathrm{mg}$ (soit $0,1 \mathrm{mCi}$ ) de suspension d'algues fuées est déposé sur le disque oral de chaque actinie. Ces cyanophycées mesurent de 4 à $8 \mu \mathrm{m}$;

Lipides, $1 \mathrm{ml}$ d'huile de foie de morue ou de pétrole (BAL 150 fourni par l'Institut français du Pétrole) est injecté dans la cavité entérique de chaque actinie.

\section{Résultats.}

D-glucose. - Après $2 \mathrm{~h}$ de chasse le marquage est intense dans la moitié apicale de l'ectoderme. Il est faible dans sa portion basale, la mésoglée et l'endoderme. Sur les coupes autoradiographiques le nombre de grains comptés dans ces zones représente en moyenne 30 p. 100 de celui observé dans la moitié apicale de l'ectoderme. Après $12 \mathrm{~h}$ de chasse l'ectoderme est uniformément marqué mais la mésoglée et l'endoderme le demeurent peu. Après $48 \mathrm{~h}$ l'intensité du marquage diminue globalement sauf dans la portion supérieure des entéroïdes. Après 6 jours le marquage esł faible sauf à la base des tractus ciliaires des entéroïdes (Photo 1) dont les cellules flagellées assurent les courants d'eau vitaux à l'actinie.

\section{L-leucine.}

a) Chez les actinies immergées, après $2 \mathrm{~h}$ de chasse, le marquage de l'ectoderme est trois fois supérieur à celui de l'endoderme. Les sécrétions des cellules glandulaires ne sont pas marquées, par contre une partie des cellules amoeboïdes de la mésoglée le sont. Après 2 jours le marquage de l'ectoderme demeure supérieur à celui de l'endoderme mais des amas de grains, de 2 à $3 \mu \mathrm{m}$ de diamètre apparaissent dans celui-ci, sans que nous ayons pu les mettre en relation avec un organite défini. La proportion de cellules de la mésoglée marquées diminue.

b) Chez les actinies ayant eu une injection dans leur cavité entérique le marquage est localisé à la portion apicale de l'endoderme après $2 \mathrm{~h}$ de chasse. Après 2 jours le marquage de l'endoderme est homogène.

Hydrolysat de protéines (acides aminés et glucides aminés).

a) Chez les actinies immergées, après $2 \mathrm{~h}$ de chasse, le marquage est ectodermique. Les sécrétions des cellules glandulaires ne sont pas marquées. Le marquage de l'endoderme est faible. Après 2 jours l'intensité du marquage diminue globalement, des amas de grains de 2 à $3 \mu \mathrm{m}$ de diamètre apparaissent dans l'endoderme sans que nous ayons pu les mettre en relation avec un organite défini. 

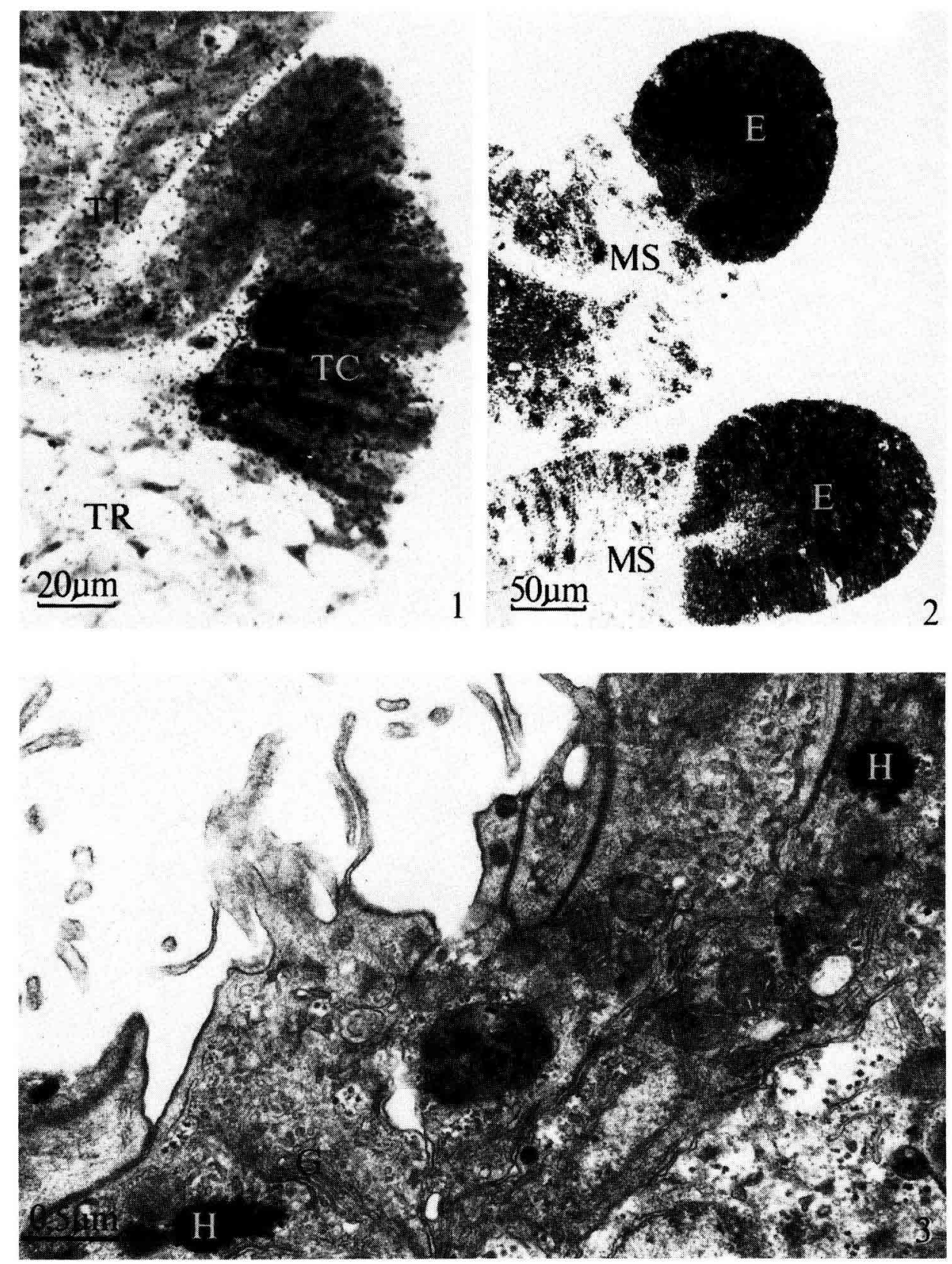

PHOTOS 1, 2, 3. 
b) Les tissus des pelotes mésentériques disséquées puis immergées sont fortement marqués au niveau des entéroïdes (Photo 2 ) mais le reste des mésentères est peu marqué après $2 \mathrm{~h}$ de chasse.

Cyanophycées. - Les algues sont visualisées sur les lames autoradiographiques sous forme d'amas de grains de 5 à $9 \mu \mathrm{m}$ de diamètre. Après $2 \mathrm{~h}$ de chasse, ceux-ci sont localisés au niveau des zones endodermiques de phagocyłose (fig. 1). Parmi ces zones celle des tractus intermédiaires des entéroïdes trilobés est particulièrement marquée. Après 6 jours, le marquage des zones de phagocytose est plus faible mais l'ensemble de l'individu en présente un diffus.

Lipides. - L'huile de foie de morue et le pétrole injectés dans la cavité entérique y sont absorbés de manière identique par les cellules phagocytaires et par certaines cellules des entéroïdes. Des tubules et vésicules apicales de $400 \AA$ de diamètre convergent vers des inclusions de 3000 à $5000 \AA$, au contenu opaque aux électrons assimilable aux lipides pinocytés (Photo 3). Lors du prolongement 1 à 9 jours des expériences de nutrition avec des lipides, ceux-ci s'accumulent à la base des cellules phagocytaires dans des inclusions qui peuvent atteindre plusieurs micromètres de diamètre ef envahir tout le cytoplasme de ces cellules. Les tractus intermédiaires des entéroïdes et la région des mésentéroïdes composée de cellules phagocyłaires prennent alors l'aspect d'un tissu adipeux.

\section{Discussion.}

Absorption des substances dissoutes.

Nos résultats confirment que l'absorption du glucose et des acides aminés dissous dans le milieu marin s'effectue essentiellement au niveau de l'ectoderme de la colonne et des tentacules. Les macromolécules, telles que la ferritine, ne sont par contre pas captées par l'ectoderme, sauf au niveau des acrorhages (Van Praët, 1978). Cette limite de taille des substances absorbées par l'ectoderme correspond à l'absence de cellule phagocytaire ectodermique ef contrairement à l'hypothèse de Schlichter (1978) à l'inexistence d'une digestion préorale. La rapidité de l'absorption ectodermique des substances dissoutes dans le milieu marin, soulignée par les précédents auteurs, ne doit pas faire négliger les capacités d'absorption de certains tissus de la cavité entérique. Le marquage intense des entéroïdes lorsque des pelotes mésentériques isolées sont directement immergées dans le milieu contenant l'hydrolysat de protéines permet d'ailleurs de compléter le schéma de la digestion extracellulaire de contact des actinies (Nicol, 1959). Lors de celle-ci les enzymes sécrétées par les cellules

PHOTO 1. - 6 jours après l'absorption de glucose ${ }^{14} \mathrm{C}$, apparition d'un marquage à la base des tractus ciliaires des entéroïdes frilobés, probablement lié au glycogène des rosettes visibles en microscopie électronique à ce niveau. TC : tractus ciliaire ; TI : tractus intermédiaire ; TR : tractus réticulaire.

PHOTO 2. - Accumulation d'acides aminés ef de glucides aminés au niveau des entéroïdes, après $2 \mathrm{~h}$ d'incubation suivies de $2 \mathrm{~h}$ de chosse. $E$ : entéroïde ; $M S$ : mésentéroïde.

PHOTO 3. - Micrographie de microscopie électronique. Absorption d'hydrocarbures (12 h d'expérience) par les cellules phagocyfaires des mésentérö̈des. G : appareil de Golgi ; H : hydrocarbures. 
à zymogène des entéroïdes fragmentent la proie en éléments de diverses tailles. Il était connu que les macromolécules et les particules sont captées par les cellules phagocytaires des mésentéroïdes, des tractus intermédiaires, de la colonne et des tentacules (Van Praët, 1978). Les acides aminés ef les glucides apparaissent pour leur part susceptibles d'être directement absorbés, d'après nos observations, par les cellules des entéroïdes (Photo l) qui enserrent étroitement la proie lors de cette phase de la digestion (fig. 1).

\section{Absorption des particules.}

Certaines des zones endodermiques captant la ferritine (Van Praët, 1978) ne phagocytent pas les cyanophycées $(4$ à $8 \mu \mathrm{m}$ ). C'est le cas des cellules à vacuole et des cellules à concrétions (Van Praët, 1977) des tractus réticulaires qui ne semblent donc capables que de pinocyter des macromolécules. Il faut noter que des images de microscopie électronique nous font considérer que les cellules à concrétions ef celles à vacuole correspondent à deux stades d'un même type cellulaire. A l'inverse les cellules phagocytaires des tractus intermédiaires sont très fortement marquées lors des expériences avec les cyanophycées. Ces tractus sont situés dans le prolongement du pharynx, sur le trajet des courants d'eau renouvelant le milieu colentérique (fig. 1). Les tractus intermédiaires semblent donc piéger efficacement les particules qui passent nécessairement à leur niveau avant d'être expulsées de la cavité entérique.

Les larves incubées par les adultes dans leur cavité entérique y bénéficient à la fois des substances dissoutes (Chia, 1972) et des particules, captées dans le milieu par le parent ou produites par sa digestion extracellulaire. Des larves au stade actinella, incubées par des individus ayant subi l'expérience avec la ferritine se sont en effet révélées avoir absorbé celle-ci au niveau de l'endoderme de leur colonne.

Les lipides pinocytés sont stockés à la base des cellules phagocytaires et ne semblent pas l'objet d'un transfert vers d'autres régions. Néanmoins des activités lipasiques sont décelables par la méthode cyłoenzymologique de Gomori à la périphérie : des inclusions apicales lors de l'absorption ef des réserves lipidiques lors du jeûne (Van Praët, à paraître).

Le transfert des substances absorbées.

Chez Actinia equina, comme chez Anemonia sulcafa (Schlichter, 1975), le transfert des acides aminés et glucides de l'ectoderme vers l'endoderme, ou vice-versa est lent, et dans le cadre de ces études nous ne pouvons pas définir les molécules où est intégrée la radioactivité révélée. Néanmoins l'observation des autoradiographies d'animaux, après différents temps de chasse permet de formuler des hypothèses sur les voies de transfert des molécules absorbées, ou de produits de leurs calabolisme, vers les diverses régions de l'actinie.

Lors des expériences avec la proline (Doumenc, 1979) et la leucine, le quart des cellules amoeboïdes de la mésoglée est sélectivement marqué, après $2 \mathrm{~h}$ de chasse. La disparition des cellules marquées dans la mésoglée après des chasses de $16 \mathrm{~h}$ (Doumenc, 1979) d̀ $48 \mathrm{~h}$ peut être analysée comme le transfert des molécules incorporées, ou de produits de leur catabolisme, vers d'autres tissus. Par contre nous n'avons pas observé de marquage spécifique de ces cellules lors de l'expérience avec le glucose, au cours des six jours qui précèdent l'apparition d'une radioactivité à la base des tractus 
ciliaires (Photo I). De nombreuses rosettes de glycogène sont visibles en microscopie électronique à ce niveau ; dans ce cas le marquage pourrait correspondre à l'incorporation d'une partie du glucose ${ }^{14} \mathrm{C}$ métabolisé dans ce glycogène. Les cellules amœboïdes de la mésoglée seraient donc selon nous susceptibles de contribuer au transfert de certaines molécules, mais une diffusion de cellule à cellule et à travers la mésoglée pourrait également exister ef assurer une partie des transferts, en particulier ceux des petites molécules glucidiques.

L'absorption par l'ectoderme des substances dissoutes dans le milieu environnant et par l'endoderme des particules et des produits de la digestion extracellulaire pourrait assurer à chacun de ces feuillets une autonomie trophique partielle. En dehors des périodes de jeûne, où sont métabolisées les réserves lipidiques, les transferts pourraient être limités à des échanges locaux de cellule à cellule.

\section{Conclusion.}

Les substances dissoutes dans le milieu marin : glucides et acides aminés, sont essentiellement absorbées par l'ectoderme. Par contre les macromolécules ne le sont pas à ce niveau. Elles sont drainées ainsi que les proies dans la cavité entérique où s'effectue la digestion.

Les macromolécules, les particules et les fragments de proie (jusqu'à une taille de quelques micromètres), captés dans le milieu ou produit par la digestion extracellulaire, sont absorbés par les cellules phagocytaires groupées dans des zones particulières de l'endoderme. Les petites molécules produites par cette digestion extracellulaire sont absorbées par les cellules des entéroïdes qui enserrent la proie.

Chez ces animaux dépourvus de système circulatoire le transfert des éléments absorbés est lent. Les cellules amœboìdes de la mésoglée pourraient intervenir dans celui de certains acides aminés et macromolécules. De plus les capacités d'absorption de tous les tissus peuvent limiter la nécessité de tels transferts.

\footnotetext{
Journées Ingestion-Digestion-Absorption de l'Association française de Nutrition, Paris, 15-16 novembre 1979.
}

\section{Références}

CHIA F. S., 1972. Note on the assimilation of glucose and glycine from sea water by the embryos of the sea-anemone, Actinia equina. Can. J. Zool., 50, 1333-1334.

DOUMENC D. A., 1979. Ełude de l'organogenèse de l'actinie ocontiaire Cereus pedunculatus Pennant. Th. Doct. Ełat, Muséum et Paris VI, 242 pP.

JØRGENSEN B. C., 1976. August Pütter, August Kroch and modern ideas on the use of dissolved organic matter in aquatic environments. Biol. Rev., 51, 291-328.

NICOL J. A., 1959. Digestion in sea anemones. J. Mar. Biol. Ass. U. K., 38, 281-297.

SCHLICHTER D., 1975. The importance of dissolved organic compounds in sea water for the nutrition of Anemonia sulcata Pennant. Proc. Eur. mar. biol. Symp., 9, 395-405.

SCHLICHTER D., 1978. On the ability of Anemonia sulcata (Coelenterata, Anthozod) to absorb charged and neutral amino acids simultaneously. Mar. Biol., 45, 97-104.

VAN PRAËT M., 1977. Les cellules à concrétions d'Actinia equina L. C. R. Acad. Sci. Paris, sér. D, 285, $45-48$.

VAN PRAËT M., 1978. Etude histochimique et ultrastructurale des zones digestives d'Actinia equina $L$. (Cnidaria, Actiniaria). Cah. Biol. Mar., 19, 415-432. 